\title{
Two-Year Outcome of Aflibercept for the Treatment of Choroidal Neovascularization in Punctate Inner Choroidopathy
}

\author{
Luis Arrevola María Almudena Acero María Jesús Peral \\ Clínica Baviera, Madrid, Spain
}

\section{Keywords}

Aflibercept · Punctate inner choroidopathy · Choroidal neovascularization · Anti-vascular endothelial growth factor $\cdot$ Ranibizumab $\cdot$ Bevacizumab

\begin{abstract}
Punctate inner choroidopathy $(\mathrm{PIC})$ is a rare inflammatory chorioretinopathy that predominantly affects young myopic women. Visual prognosis is generally good, but occurrence of choroidal neovascularization (CNV) is common and may be vision threatening. Case reports and short case series support the effectiveness of intravitreal anti-vascular endothelial growth factor (VEGF) agents (ranibizumab and bevacizumab) for CNV associated with PIC given their anti-angiogenic and anti-inflammatory effects. Evidence concerning aflibercept, a more recent intravitreal anti-VEGF, is limited to a single case report. In this case report, we illustrate the case of a 43-year-old myopic woman presenting with visual acuity loss and distortion in the right eye over the last 5 days in whom CNV associated with PIC was diagnosed. Treatment with 1 injection per month of intravitreal aflibercept for 2 months and full-dose oral prednisone for 1 week, being tapered afterwards, improved visual acuity and resolved CNV, with benefits lasting up to 24 months.




\section{Introduction}

Punctate inner choroidopathy (PIC) is a rare inflammatory chorioretinopathy characterized by multiple, small, round, yellow-white punctate lesions seen on fundoscopy, apparently in the absence of intraocular inflammation [1, 2]. PIC predominantly affects young myopic women $[1,2]$. Symptoms of PIC typically include loss of central visual acuity (VA), central scotomas, and photopsias [1]. Visual prognosis is generally good [1,3], and most patients do not need treatment [1,2]. Choroidal neovascularization (CNV) is one of the most common visionthreatening complications in PIC [1]. Incidence rates up to 69-75\% have been reported either at initial presentation or as early as within 1 year [1,3], although CNV may occur years after the initial lesions [4]. CNV requires immediate care [2].

Treatment modalities, such as systemic and local steroids or other immunosuppressant agents, laser photocoagulation, photodynamic therapy, and submacular surgery, commonly used to manage subfoveal CNV in patients with PIC, have shown several limitations [1,2,4]. In recent years, the potential of anti-vascular endothelial growth factor (VEGF) therapy has been the focus of research for $\mathrm{CNV}$ associated with PIC given its anti-angiogenic and anti-inflammatory effects. Evidence on the benefits provided by anti-VEGF therapies (mainly ranibizumab and bevacizumab) arises from small case series (summarized by Campos et al. [1] up to 2014), which limits the evidence necessary to allow appropriate treatment choice. Of these, only ranibizumab is licensed for intravitreal use in the treatment of neovascular age-related macular degeneration (AMD) and other eye pathologies characterized by ocular neoangiogenesis.

Aflibercept is a more recent anti-VEGF agent licensed for the treatment of neovascular (wet) AMD and visual impairment due to myopic CNV or macular edema secondary to retinal vein occlusion or diabetic macular edema. The affinity of aflibercept for VEGF-A and placental growth factor is much higher than that of ranibizumab and bevacizumab [5]. Moreover, its longer intravitreal half-life translates into a need for less frequent injections [5], as evidenced in the VIEW 1 and VIEW 2 studies versus ranibizumab, either when used at fixed doses or in a pro-re nata (PRN) regimen (96-week extension) in patients with neovascular AMD [6]. Evidence of its benefit in the treatment of non-AMD-related CNV is growing but still sparse. Only 1 case report about the benefit of aflibercept in CNV associated with PIC has been published so far [7].

\section{Case Report}

We report the case of a 43-year-old myopic woman who presented with loss of VA and distortion in the right eye (OD) for 5 days and who was diagnosed with CNV associated with PIC. Treatment with once-monthly intravitreal injections of aflibercept for 2 months and oral prednisone for 1 month improved VA and resolved CNV, with visual and anatomic benefits lasting up to 24 months.

\section{Results}

A 43-year-old healthy female presented with a 5-day history of visual deterioration, distorted central vision, and scotomata OD. Ophthalmic history included LASIK surgery for $-6 \mathrm{D}$ myopia with astigmatism in both eyes (OU) performed 18 years ago. The patient reported 
having experienced transient episodes of blurred vision OU during the past 2 years for which she had not had an ophthalmologic evaluation. Best-corrected visual acuity (BCVA) at presentation was 20/25 OD and 20/20 in the left eye. Funduscopy revealed small yellowish-white spots in the nasal choroid up to the optic nerve OU, with no signs of inflammation in the vitreous or anterior chamber. Serous retinal detachment suggestive of CNV was also observed OD (Fig. 1a). Fluorescein angiography (FA) showed multiple hyperfluorescent spots OU in the early phases that increased slightly in the later phases of the angiogram. Early-phase FA showed multiple retinal pigment epithelial window defects OU corresponding to scars. In OD, a hyperfluorescent superotemporal area was observed, with dye leakage from one of the spots that suggested the presence of classic CNV associated with an active inflammatory lesion. Unfortunately, electronic records of these FA images are currently unavailable due to IT problems. Central spectral-domain optical coherence tomography (SD-OCT) showed a retinal pigment epithelial elevation above Bruch's membrane, together with increased central retinal thickness, which confirmed CNV. A small amount of subfoveal fluid was seen (Fig. 1b). The patient underwent systemic evaluation by an internal medicine specialist to investigate possible causes for her eye condition (e.g., autoimmune or infectious diseases). One week later, the patient came to our office complaining of worsening scotomata OD. Autofluorescence (AF) showed chronic inactive lesions (hypoautofluorescent spots) OU and a hyperautofluorescent spot corresponding to an active inflammatory lesion OD (Fig. 2a). The FA showed an increase of dye leakage and hyperfluorescence in the late phases, which confirmed the presence of active CNV arising from a typical inflammatory PIC lesion (Fig 2b). SD-OCT revealed an increase in the size of the lesion and increased leakage, with exudative cystoid spaces (Fig. 2c) and subretinal fluid (Fig. 2d). Blood tests showed elevation of IgG to Epstein-Barr virus. All these findings led to a diagnosis of PIC OU with an active inflammatory lesion associated with CNV OD.

After discussing various treatment options and obtaining informed consent for off-label use, in September 2016 the patient was started on intravitreal aflibercept $2.0 \mathrm{mg} / 0.05 \mathrm{~mL}$ OD with the intention to use a complete loading dose ( 1 injection per month for 3 months). Oral prednisone ( $1 \mathrm{mg} / \mathrm{kg} /$ day) was added for 1 week, being subsequently tapered every $4-5$ days and finally stopped after 1 month. The patient reported a remarkable improvement after the first 2 intravitreal injections of aflibercept. BCVA OD had increased to 20/25+4, and multimodal imaging with FA and AF showed remission of the inflammatory and CNV lesions OD, with CNV appearing as mild hyperautofluorescence on the AF image, similar to the scars of the old inflammatory lesions (Fig. 3a). No dye leakage was observed on FA (Fig. 3b). SD-OCT showed disappearance of subfoveal fluid and the presence of residual fibrotic scar tissue at the place of the CNV (Fig. 3a-c). In agreement with the patient, aflibercept was stopped and regular bimonthly controls were scheduled. VA remained stable during the subsequent evaluations (until February 2017) and during the next follow-up visits taking place at 3 and 8 months (May and November 2017). At the moment of writing this article, the last visit was conducted 1 month ago (July 2018). Total follow-up since the first intravitreal injection of aflibercept has been 24 months. No adverse events have been reported during this period.

\section{Discussion/Conclusion}

This case report illustrates the benefits of aflibercept in the resolution of CNV associated with PIC in a 43-year-old woman. Moreover, benefits were apparent after 2 single monthly doses and lasted during the 2 years of follow-up. 
Due to the inflammatory nature of the disease and given the good visual prognosis - unless complicated by CNV or subretinal fibrosis - $[1,3]$, the patient suffered only transient episodes of blurred vision OU in the past 2 years which seemed not to affect daily life and for which she did not request an ophthalmic consultation. Exacerbation of the typical symptoms of PIC and visual complications (vision deterioration, distorted central vision, and scotomata) $\mathrm{OD}$, likely as a consequence of the development of CNV in this eye, caused the patient to visit our office. Typical signs of choroiditis on funduscopy made us suspect the presence of PIC, especially given the bilateralism and the fact that the patient was a young myopic woman. The presence of antibodies against Epstein-Barr virus, which have been associated with the development of this rare inflammatory chorioretinopathy [8], supported this hypothesis, which was subsequently confirmed by imaging findings on AF and FA. CNV OD was also detected on these studies. Development of CNV within the first year after the initial lesions occur is common $[1,3,4]$. Unfortunately, our patient only sought medical attention when the effects of CNV were already apparent, which may reflect a common situation in this disease. CNV occurrence requires immediate care [2].

Several case reports and short case series have provided evidence of the benefits of antiVEGF therapy for treating CNV associated with PIC, both with and without concomitant treatment (i.e., oral corticoids) to manage inflammatory lesions. These studies have been mainly conducted with ranibizumab [1,9-12], one of the first anti-VEGF agents licensed for intravitreal use, but also with bevacizumab [13-15], which is often used off-label in intravitreal injections. Given the lack of guidance regarding how to best use these anti-VEGF agents in offlabel conditions, there is a lack of uniformity in the different reports. Hence, some studies report using an initial loading dose ( 1 injection every 4 weeks for 3 months) of bevacizumab [13] or ranibizumab [10] with 6 or 12 months of follow-up, respectively. Other studies report the effectiveness of a single injection of either ranibizumab [9] or bevacizumab [15] with visual and anatomic benefits lasting during 8 and even 24 months of follow-up, respectively. Other studies have assessed the use of these anti-VEGF agents in PRN regimens, also reporting visual and anatomic benefits during with follow-ups of up to 1.5 years [12,14]. Our approach was to use a loading dose of aflibercept, together with 1-week treatment with oral prednisone, gradually tapering off as reported by other authors [1]. In agreement with the patient, who showed remarkable improvement of VA, treatment with aflibercept was stopped after 2 doses when evidence of remission of the CNV lesion was found on AF and FA.

In the only case published so far in which aflibercept has been used, improvement of VA from $2 / 10$ to $7 / 10$ was reported 5 months after starting the loading dose. Despite the authors' claim that no further treatment was needed during the regular visits, no information about the follow-up is given. Our report is thus the first one showing the effectiveness and safety of aflibercept for the treatment of CNV associated with PIC during a long-term follow-up (up to 2 years). The possibility that a single dose would have been enough - as has been reported in 2 cases in which ranibizumab or bevacizumab were used $[9,15]$ - cannot be ruled out.

\section{Conclusion}

This case report demonstrates a long-lasting favorable therapeutic outcome (up to 2 years) when using aflibercept for CNV associated with PIC, with remission of the lesion and improvement of visual outcomes after 2 injections. 
Acknowledgements

The authors thank Beatriz Viejo, PhD, for her assistance in the writing of the manuscript and editorial support.

\section{Statement of Ethics}

The authors have no ethical conflicts to disclose.

\section{Disclosure Statement}

The authors have no conflicts of interest to disclose.

\section{Funding Sources}

The writing of this manuscript was supported by Bayer Hispania, who had no influence on the interpretation of results.

\section{References}

1 Campos J, Campos A, Mendes S, Neves A, Beselga D, Sousa JC. Punctate inner choroidopathy: a systematic review. Med Hypothesis Discov Innov Ophthalmol. 2014;3(3):76-82.

2 Amer R, Lois N. Punctate inner choroidopathy. Surv Ophthalmol. 2011 Jan-Feb;56(1):36-53.

3 Channa R, Ibrahim M, Sepah Y, Turkcuoglu P, Lee JH, Khwaja A, et al. Characterization of macular lesions in punctate inner choroidopathy with spectral domain optical coherence tomography. J Ophthalmic Inflamm Infect. 2012 Sep;2(3):113-20.

4 Brouzas D, Charakidas A, Rotsos T, Moschos MM, Loukianou H, Koutsandrea C, et al. Choroidal neovascularization due to punctate inner choroidopathy: long-term follow-up and review of literature. Clin Ophthalmol. 2010 Aug;4:871-6.

5 Stewart MW, Rosenfeld PJ, Penha FM, Wang F, Yehoshua Z, Bueno-Lopez E, et al. Pharmacokinetic rationale for dosing every 2 weeks versus 4 weeks with intravitreal ranibizumab, bevacizumab, and aflibercept (vascular endothelial growth factor Trap-eye). Retina. 2012 Mar;32(3):434-57.

6 Schmidt-Erfurth U, Kaiser PK, Korobelnik JF, Brown DM, Chong V, Nguyen QD, et al. Intravitreal aflibercept injection for neovascular age-related macular degeneration: ninety-six-week results of the VIEW studies. Ophthalmology. 2014 Jan;121(1):193-201.

7 Kabanarou SA, Boutouri E, Xirou T, Vasilias D, Gkizis I, Chatziralli I. Intravitreal aflibercept for choroidal neovascularization secondary to punctate inner choroidopathy. Presented at: The 9th Annual Congress on Controversies in Ophthalmology: Europe (COPHy EU), Athens (Greece), Abstract 19.2018. Available from: http://www.comtecmed.com/cophy/2018/Uploads/Editor/Abstracts/19.pdf

8 Tiedeman JS. Epstein-Barr viral antibodies in multifocal choroiditis and panuveitis. Am J Ophthalmol. 1987 May;103(5):659-63.

9 Leung AK, Weisbrod DJ, Schwartz C. Intravitreal ranibizumab in the treatment of choroidal neovascular membrane secondary to punctate inner choroidopathy. Can J Ophthalmol. 2010 Jun;45(3):300-1.

10 Jiménez B, Pinilla I, Cristóbal JA, Mínguez E, Pérez D, Cruz N, et al. [Intravitreal ranibizumab in the treatment of subretinal neovascularization in a case of punctate inner choroidopathy]. Arch Soc Esp Oftalmol. 2014 Mar;89(3):130-2.

11 Roca M, Menezo M, Ronchera JM, Esteban JM, Roca B. Punctate inner choroidopathy complicated with exudative neurosensory detachment: A favourable response to treatment with systemic corticosteroids and intravitreal ranibizumab. Arch Soc Esp Oftalmol. 2017 Jul;92(7):343-6.

12 Rouvas A, Petrou P, Douvali M, Ntouraki A, Vergados I, Georgalas I, et al. Intravitreal ranibizumab for the treatment of inflammatory choroidal neovascularization. Retina. 2011 May;31(5):871-9. 


\section{Case Reports in Ophthalmology}

13 Chan WM, Lai TY, Liu DT, Lam DS. Intravitreal bevacizumab (avastin) for choroidal neovascularization secondary to central serous chorioretinopathy, secondary to punctate inner choroidopathy, or of idiopathic origin. Am J Ophthalmol. 2007 Jun;143(6):977-83.

14 Zhang H, Liu ZL, Sun P, Gu F. Intravitreal bevacizumab as primary treatment of choroidal neovascularization secondary to punctate inner choroidopathy: results of a 1-year prospective trial. Retina. 2012 Jun;32(6):1106-13.

15 Campos J, Campos A, Beselga D, Mendes S, Neves A, Sousa JP. Punctate inner choroidopathy: a clinical case report. Case Rep Ophthalmol. 2013 Sep;4(3):155-9.
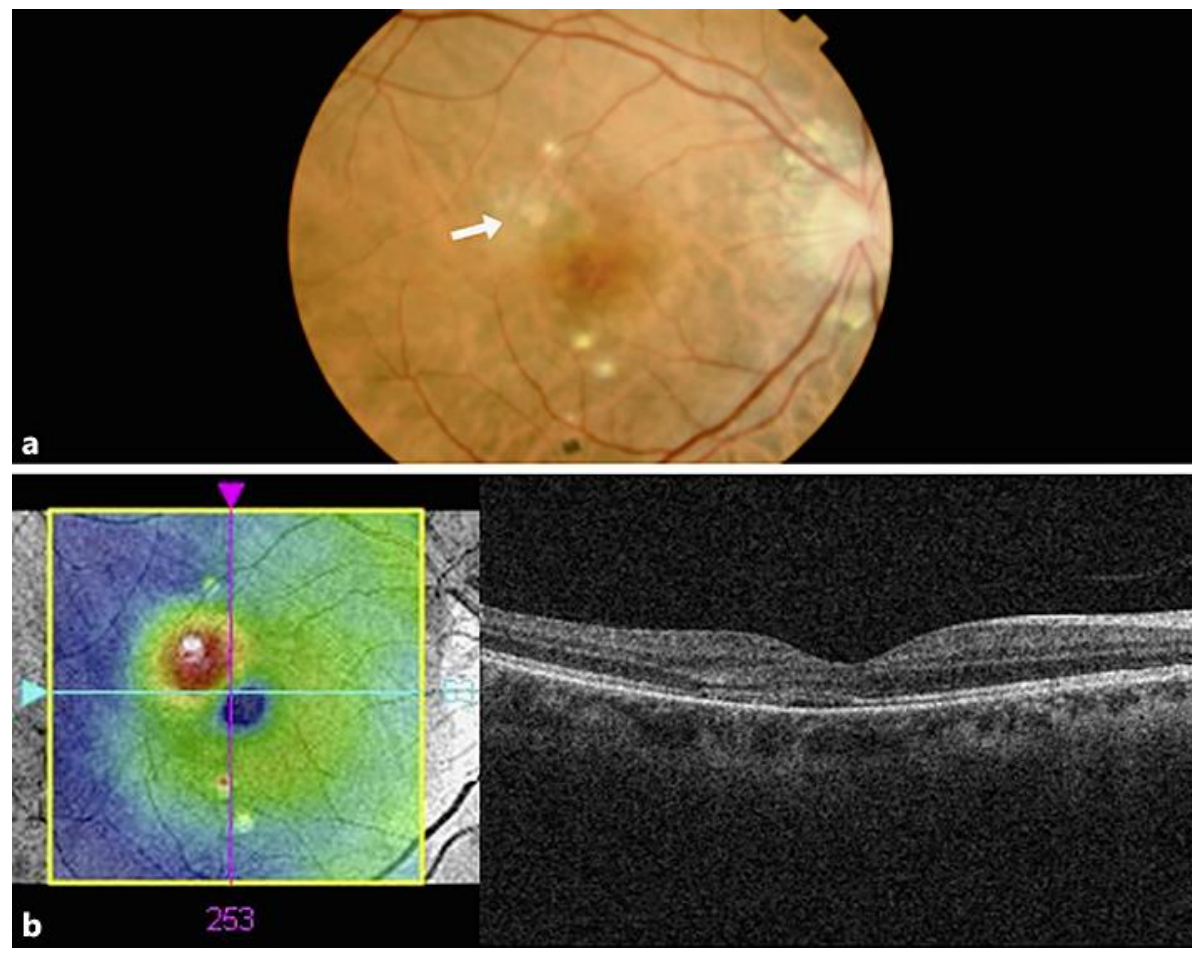

Fig. 1. a Funduscopy showing small yellowish-white spots at the choroid and serous detachment (only OD is shown) at the first visit. b Central spectral-domain optical coherence tomography of OD performed on the same day showing retinal pigment epithelial elevation above Bruch's membrane together with increased central retinal thickness. A small amount of subretinal fluid was observed (dark stain). OD, right eye. 


\section{Case Reports in Ophthalmology}
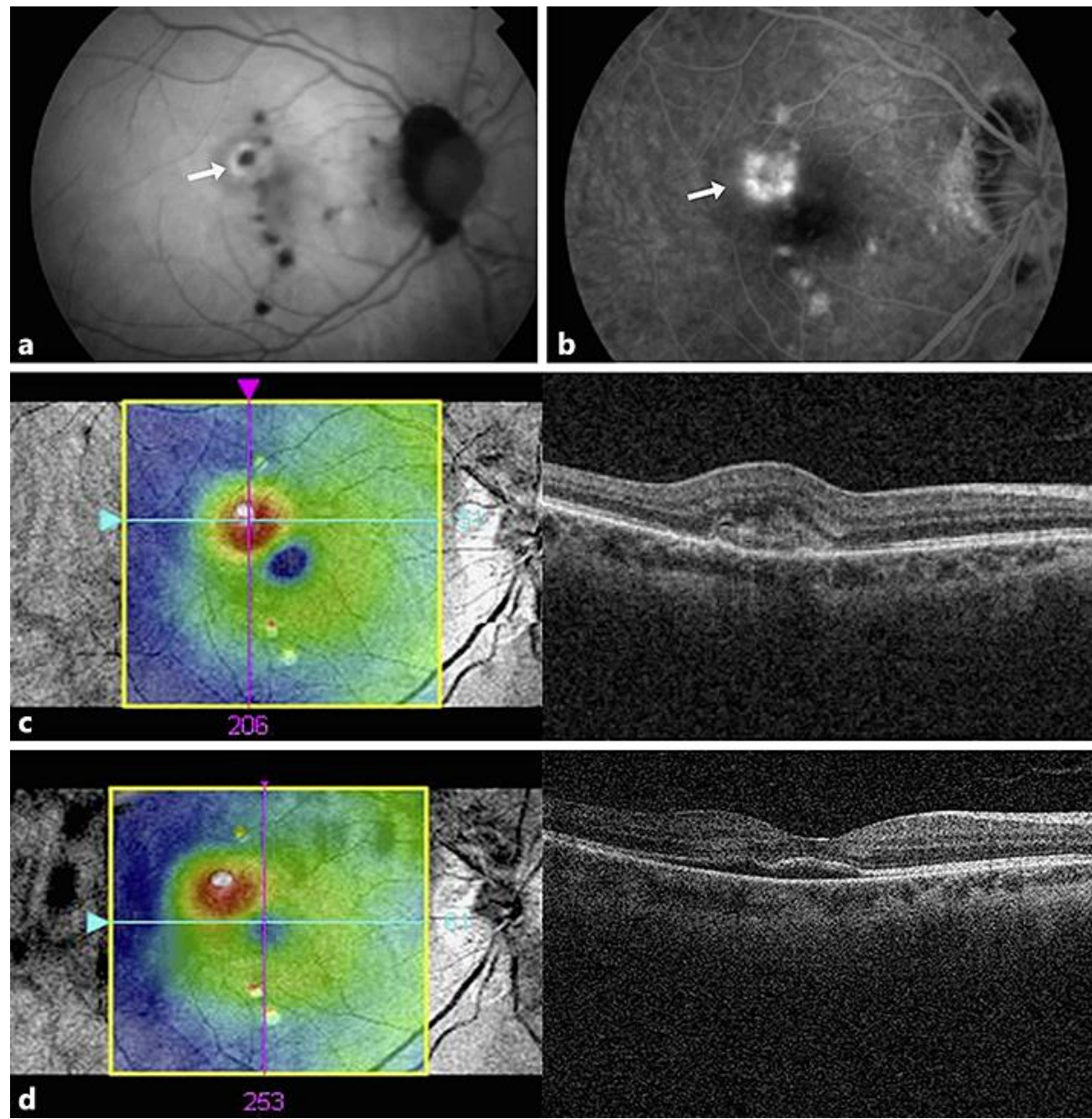

Fig. 2. a Autofluorescence OD showing chronic inflammatory lesions (hypoautofluorescent spots) and an active inflammatory lesion (hyperautofluorescent spot). b Fluorescein angiography OD showing an area of hyperfluorescence due to an increase of dye retention in the early phases that persisted in the late phases. c SD-OCT of the superotemporal quadrant of the OD showing augmentation of the lesion size and an increase of leakage with exudative cystoid spaces. $\mathbf{d}$ Central SD-OCT OD showing the presence of subretinal fluid. All images were taken 1 week after the first visit. OD, right eye; SD-OCT, spectral-domain optical coherence tomography. 


\section{Case Reports in Ophthalmology}
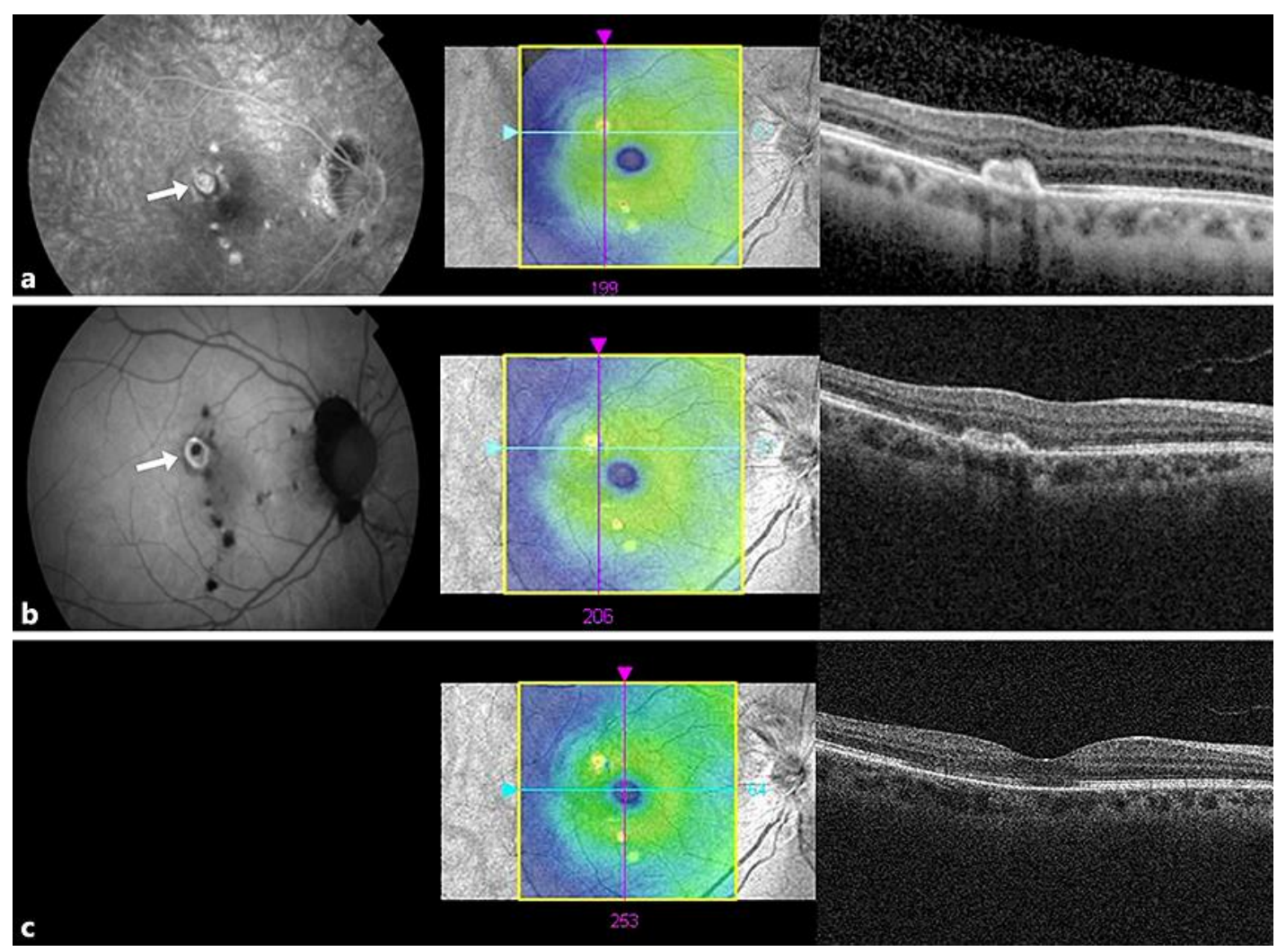

Fig. 3. Multimodal image (autofluorescence + SD-OCT [a] and fluorescein angiography + SD-OCT [b]) OD showing remission of the inflammatory and CNV lesions after 2 intravitreal injections of aflibercept. c SDOCT of the superotemporal quadrant performed on the same day also showing disappearance of subretinal fluid compared to Figure 2d. OD, right eye; SD-OCT, spectral-domain optical coherence tomography. 